Ann. Biol. anim. Bioch. Biophys., I970, 10 (4), 659-677.

\title{
ÉVOLUTION DE LA SÉCRÉTION DES ACIDES GRAS DES MATIERES GRASSES DU LAIT AU COURS DE LA LACTATION DE LA VACHE
}

\author{
C. DECAEN* et J. ADDA** \\ avec la collaboration technique de Renée Lefaivre, B. Marquis et A. Hoden \\ * Station de Recherches sur l'Élevage des Ruminants, \\ Centre de Recherches de Clermont-Ferrand, 63-Saint-Genès-Champanelle \\ ** Station centrale de Recherches laitières et de Technologie des Produits animaux, \\ Centre national de Recherches zootechniques, 78 -Jouy-en-Josas \\ Institut national de la Recherche agronomique
}

\begin{abstract}
RÉSUMMÉ
Nous avons observé l'évolution qualitative et quantitative de la sécrétion des principaux acides gras des triglycérides du lait de 17 vaches, au cours d'une lactation de 40 semaines. Ces vaches, alimentées individuellement à l'étable, avec une ration de foin de luzerne distribuée sous forme longue et condensée, appartenaient à deux lots : les unes vêlant en hiver à Jouy, les autres en automne à la Minière.

$\mathrm{Au}$ cours des deux premiers mois de lactation, la proportion des acides gras courts des triglycérides du lait $\left(\mathrm{C}_{4}-\mathrm{C}_{14}\right)$ augmente, celle des acides gras longs $\left(\mathrm{C}_{18}\right)$ diminue et celle de l'acide palmitique varie peu (tabl. 2) ; la quantité sécrétée d'acides gras courts augmente de 50 p. roo, celle d'acides gras longs diminue de $4^{\circ} \mathrm{p}$. Ioo et celle d'acide palmitique diminue légèrement (tabl. 3 ). Pendant ce temps, la Vache augmente ses quantités d'aliments ingérées, mais perd du poids car son bilan énergétique est très déficitaire dans les 2 ou 3 semaines qui suivent la mise bas. La baisse du taux butyreux au cours des 2 premiers mois de la lactation vient de la diminution de la sécrétion des acides gras longs du lait, elle-même liée à l'intensité de la mobilisation des réserves corporelles.

Après le $3^{\mathrm{e}}$ mois de lactation, les quantités sécrétées de lait et de matières grasses diminuent, mais la composition en acides gras des triglycérides du lait ne varie pas, bien que le taux butyreux augmente.

La production laitière semble plus stimulée en hiver et au printemps, la remontée du taux butyreux au cours de la $2^{\mathbf{e}}$ moitié de la lactation est surtout marquée à partir du mois d'août, que les vaches vêlent en novembre ou en mars.
\end{abstract}

\section{IN'TRODUCTION}

Il est bien établi que la sécrétion de matières grasses du lait de vache varie au cours de la lactation : le taux butyreux diminue au cours des 6 semaines qui suivent le vêlage, puis augmente plus ou moins rapidement par la suite ; la quantité de matières 
grasses sécrétée augmente au cours des deux premières semaines de la lactation (JARrige et Journet, I959; Decaen, Journet et Poutous, I970) puis diminue régulièrement jusqu'au tarissement. Peu d'auteurs, néanmoins, ont étudié l'influence du stade de lactation sur les caractéristiques chimiques de ces matières grasses : Kuzdzal-Savore (I956) a observé une baisse de l'indice d'iode, au cours des 4 premiers mois de la lactation, BALDWIN et LONGENECKER (I944), ANANTAKRISHNAN et al. (I946), Hosogar (I963), ADDA (I964) ont montré que les triglycérides du colostrum sont plus pauvres en acides gras à courte chaîne que ceux du lait; STuLL et al. (I966) ont décrit, au moyen d'une série d'équations, les variations de la composition en acides gras de la matière grasse du lait de 4 vaches pendant toute une lactation.

Nous nous sommes attachés pour notre part à décrire sous le double aspect quantitatif et qualitatif 1'évolution de la sécrétion des acides gras des matières grasses du lait de vache, au cours des ro mois de lactation, en limitant les variations d'origine alimentaire par la distribution du même type de ration aux animaux durant toute leur lactation. Une première partie des résultats a été publiée au XVIII Congrès International de Laiterie de Munich (DECAEN et ADDA, I966).

\section{MATÉRIEL ETT MÉTHODES}

\section{Animaux}

L'étude a porté sur 2 lots de vaches du troupeau du C. N. R. Z. L'un comprend 9 animaux qui ont vêlé en hiver (février-mars 1964) à l'étable de Jouy-en-Josas, l'autre, 8 animaux qui ont vêlé à l'automne (octobre à décembre 1964) à l'étable de la Minière.

Le tableau I donne les caractéristiques des animaux (race, âge, numéro de lactation, date du vêlage) et met en regard les résultats de la lactation expérimentale (quantité de lait, taux butyreux, taux de matière azotée) de ceux de la lactation précédente.

\section{Condition de vie et alimentation}

Les animaux ont été maintenus en stabulation entravée et ont reçu individuellement et à volonté, le matin, du foin de luzerne sous forme longue et le soir, du foin de luzerne condensé (broyé à la grille de $3 \mathrm{~mm}$ et aggloméré en bouchons de $10 \mathrm{~mm}$ de diamètre). Cette ration fut complémentée par un aliment concentré comprenant $85 \mathrm{p}$. Ioo de céréales dont $60 \mathrm{p}$. Ioo d'orge, distribué en quantité suffisante pour que soient couverts les besoins nutritifs évalués chaque semaine pour chaque animal, compte tenu de son poids et de sa production laitière.

Sur le plan sanitaire, nous avons observé quelques cas de perturbations métaboliques au cours des I5 premiers jours de la lactation surtout à l'étable de Jouy où les animaux furent moins bien préparés au nouveau régime à base de foin condensé qu'à la Minière.

\section{Échantillonnage et analyse}

Les quantités individuelles et journalières d'aliment ingérées, exprimées en kilogrammes de matière sèche ont été estimées par pesées des quantités offertes et refusées. A partir de ces résultats, connaissant la valeur nutritive des aliments distribués et les besoins théoriques des vaches, nous avons estimé le bilan énergétique de chaque animal, chaque semaine, en considérant la différence de l'énergie ingérée et de l'énergie excrétée. Par ailleurs, nous avons mesuré le poids vif des animaux par une pesée hebdomadaire.

La quantité individuelle de lait a été pesée à chacune des deux traites ; la teneur en matières grasses a été mesurée chaque jour, par la méthode Gerber, sur un échantillon de lait représentatif des deux traites, et la quantité de matière grasse sécrétée par jour en a été déduite. 
La teneur en matières azotées a été également mesurée, une fois par semaine pour chaque animal, sur un échantillon individuel pondéré sur 4 traites consécutives, par la méthode colorimétrique au Noir amido (Posthumus, 1959).

\section{TABLEAU I}

Caractéristiques et résultats des lactations expérimentales comparés à ceux des lactations antérieures

(H : Française Frisonne Pie Noire, N : Normande, P. R. : Pje Rouge)

\begin{tabular}{|c|c|c|c|c|c|c|c|c|c|}
\hline \multirow[b]{2}{*}{ Vache no } & \multirow[b]{2}{*}{ Race } & \multicolumn{5}{|c|}{ Lactation expérimentale (280 jours) } & \multicolumn{3}{|c|}{ Lactation antérieure $(280 \mathrm{j})$} \\
\hline & & $\begin{array}{l}\text { Date de } \\
\text { vêlage }\end{array}$ & N. L. & Lait & T. B. & T. M. A. & Lait & T. B. & T. M. A. \\
\hline 241 & $\mathrm{H}$ & $29-03-64$ & 7 & 4610 & 31 & 35 & 4830 & 34 & 34 \\
\hline 248 & $\mathbf{H}$ & $15-03-64$ & 8 & 5060 & 36 & 32 & 4210 & 39 & 31 \\
\hline 7214 & II & $07-02-64$ & 4 & 4490 & 34 & 32 & 6000 & 36 & 31 \\
\hline 7486 & $\mathrm{~N}$ & $25-02-64$ & 4 & 5020 & 39 & 39 & 4120 & 42 & 38 \\
\hline 8129 & $\mathrm{H}$ & 21-03-6x & 3 & 3530 & 35 & 33 & 4590 & 37 & 32 \\
\hline 8279 & $\mathrm{H}$ & $09-02-64$ & 3 & 5600 & 31 & 30 & 4520 & 36 & 32 \\
\hline 9106 & $\mathrm{H}$ & $07-03-64$ & 2 & 3050 & 36 & 34 & 3260 & 37 & 33 \\
\hline 0009 & $\mathrm{H}$ & $01-02-64$ & 2 & 5640 & 37 & 35 & 4910 & 39 & 35 \\
\hline 0103 & $\mathrm{H}$ & $06-03-64$ & 2 & 4940 & 33 & 31 & 3800 & 35 & 34 \\
\hline $\begin{array}{l}\text { Lot I } \\
\text { Jouy }\end{array}$ & & 1 er mars 64 & 3,9 & 4660 & 34,7 & 33,4 & 4470 & 37,2 & 33,3 \\
\hline 9100 & $\mathrm{~N}$ & $07-10-64$ & 3 & 3210 & 36 & 34 & 3350 & 41 & 36 \\
\hline 91.66 & $\mathrm{H}$ & $01-11-6^{\prime}$ & 3 & 4250 & 36 & 34 & 3850 & 40 & 34 \\
\hline 1354 & P. R. & $22-11-64$ & 2 & 2870 & $3 \prime$ & 35 & 2780 & $3 t$ & 31 \\
\hline 9546 & $\mathrm{H}$ & $11 \cdot 11-64$ & 3 & 5350 & 38 & 34 & 5360 & 41 & 33 \\
\hline 1162 & $\mathrm{H}$ & $09-11-64$ & 1 & 4250 & 37 & 33 & - & - & - \\
\hline 0145 & $\mathrm{H}$ & $16-11-64$ & 3 & 3720 & 35 & 34 & 4100 & 40 & 32 \\
\hline 388 & $\mathrm{H}$ & $17-12-64$ & 7 & 3260 & 34 & 33 & 3700 & 37 & 33 \\
\hline 0768 & P. R. & $10-12-64$ & 3 & 4200 & 37 & 35 & 3630 & 43 & 35 \\
\hline $\begin{array}{l}\text { Lot II } \\
\text { Minière }\end{array}$ & & 15 nov. 64 & 3,1 & 3890 & 35,9 & 34,0 & 3820 & 39,4 & 33,4 \\
\hline Moyenne & & & 3,5 & 4300 & 35,2 & 33,7 & 4190 & 38,2 & 33,4 \\
\hline
\end{tabular}

En vue du dosage des acides gras des triglycérides du lait, nous avons prélevé un échantillon pondéré de 2 à $3 \mathrm{~kg}$ de lait, sur 4 traites consécutives, une fois par semaine, au cours des six premières semaines après le vêlage, puis toutes les deux semaines pendant le reste de la lactation. Après écrèmage du lait, la crème refroidie a été transformée en beurre qui a été conservé à - I $5^{\circ} \mathrm{C}$. Au moment de l'analyse, l'huile de beurre est séparée par centrifugation du beurre fondu à $70^{\circ} \mathrm{C}$ ( $3000 \mathrm{t} / \mathrm{mn}$ pendant $5 \mathrm{mn}$ ). La composition en acides gras des triglycérides du lait est déterminée en 2 étapes, par chromatographie en phase gazeuse des esters méthyliques et butyliques, préparés par transestérification de l'huile de beurre (CLÉmENT et BEZARD, 196I).

Nous avons utilisé un appareil Aerograph, modèle A $35^{\circ}$, muni d'un détecteur à catharomètre dont le signal est transmis à un enregistreur Honeywell équipé d'un intégrateur à disque. Pour les esters méthyliques, on a utilisé des colonnes de 3 mètres, remplies de Chromosorb W (6o/8o mesh) chargées à 20 p. Ioo de succinate de diéthylène glycol et maintenues à une température constante de $185^{\circ} \mathrm{C}$. Les esters butyliques ont été séparés sur des colonnes de 1,5 mètre chargées d'Apiezon $\mathrm{L}$ et chauffées à $2 \mathrm{I}^{\circ} \mathrm{C}$. Le gaz vecteur dont le débit était d'environ 3 litres par heure a été l'hélium dans les deux cas. 
La volatilité des esters méthyliques est une source d'erreur importante pour la détermination quantitative des acides dont le nombre d'atomes de carbone est inférieur à ıo. C'est pourquoi nous avons procédé en deux étapes, retenant les résultats de l'analyse des esters butyliques pour ces acides courts et les résultats de l'analyse des esters méthyliques pour ceux dont la chaîne contient plus de Io atomes de carbone. Le pic correspondant à l'acide caprylique, qui figure sur les deux chromatogrammes, nous a permis d'exprimer ensuite tous les résultats en pourcentages pondéraux d'esters méthyliques. Nous n'avons pas utilisé de coefficients de correction.

Les matières grasses du lait étant pour 98 p. Ioo constituées de triglycérides (MAIsson, I962) et le poids moléculaire d'un triglycéride égal à $4 \mathrm{~g}$ près à la somme des poids moléculaires des 3 esters méthyliques correspondants, le poids de matières grasses sécrétées a été assimilé au poids du mélange d'esters méthyliques qui leur correspondent. Ainsi, on a obtenu une estimation de la production journalière d'un acide gras en multipliant le poids de matières grasses sécrétées par le pourcentage pondéral de l'ester méthylique correspondant à cet acide gras.

Pour faciliter l'exploitation des résultats, les acides gras ont été regroupés de façon un peu arbitraire, en 4 fractions, à savoir :

${ }^{\circ}$ les acides gras courts : (en $\mathrm{C}_{6}, \mathrm{C}_{8}, \mathrm{C}_{10}, \mathrm{C}_{12}$ et $\mathrm{C}_{14}$ ) qui seraient synthétisés en majeure partie à partir de l'acétate et du $\beta$-hydroxybutyrate circulant dans le sang;

$2^{0}$ les acides gras longs : stéarique et oléique dont l'origine principale serait les acides gras préformés dans le sang; nous n'avons pas dans ce groupe les acides gras polyinsaturés, vu la précision assez médiocre de leur détermination.

$3^{\circ}$ 1'acide palmitique, constituant principal qui semble être issu des 2 sources à la fois ;

$4^{0}$ les acides gras mineurs des groupes $C_{14: 1}-C_{15: 1}$ dont les pics sur le chromatogramme apparaissent entre ceux des acides myristique et palmitique.

\section{RÉSULTATS}

\section{Evolution moyenne au cours de la lactation}

\section{Consommation d'aliments -- Production laitière.}

Au cours des 40 semaines de la lactation, la quantité journalière moyenne de matière sèche ingérée augmente d'abord, passant de $\mathrm{I} 2,6 \mathrm{~kg}$ à plus de $20 \mathrm{~kg}$ au cours des sept premières semaines, puis se stabilise et enfin, à partir du $3^{e}$ mois diminue légèrement jusqu'au tarissement où elle atteint $\mathrm{I} 7,5 \mathrm{~kg}$ : la consommation d'aliment concentré augmente passant de 2,6 à 7,0 kg durant les deux premiers mois, puis diminue régulièrement pour être nulle en $35^{\mathrm{e}}$ semaine, celle de foin condensé croît durant toute la lactation de $6 \mathrm{~kg}$ à $12,5 \mathrm{~kg}$ de même que celle de foin long qui passe de 3.5 à $7,0 \mathrm{~kg}$. Durant les I 5 jours qui suivent le vêlage, alors que les besoins énergétiques sont maximum, les quantités ingérées sont faibles : il en résulte une sous-alimentation qui va en diminuant au cours des 7 premières semaines de lactation; ensuite le bilan énergétique moyen des animaux est sensiblement équilibré. Durant les 5 premières semaines, les vaches perdent $30 \mathrm{~kg}$ de poids vif en moyenne; mais elles regagnent $\mathrm{I} 8 \mathrm{~kg}$ dans les deux mois qui suivent, puis $30 \mathrm{~kg}$ en fin de lactation.

Sur l'ensemble des 40 semaines de la lactation expérimentale, les vaches ont produtit en moyenne $4300 \mathrm{~kg}$ d'un lait à $35,2 \mathrm{~g} / \mathrm{kg}$ de matières grasses et à $33,7 \mathrm{~g} / \mathrm{kg}$ de matières azotées : par rapport à la lactation précédente, les résultats moyens de quantité de lait et de teneur en matières azotées sont sensiblement les mêmes, sinon 
légèrement supérieurs, mais le taux butyreux est inférieur de $3 \mathrm{~g} / \mathrm{kg}$ (tabl. I). La quantité journalière de lait, maximum 4 semaines après le vêlage, diminue ensuite assez régulièrement tout au long de la lactation ; le taux butyreux diminue rapide ment de 47,0 à $32,5 \mathrm{~g} / \mathrm{kg}$ au cours des 7 premières semaines de la lactation, puis se stabilise jusqu'à la $20^{\mathrm{e}}$ et enfin augmente régulièrement pour atteindre $39 \mathrm{~g} / \mathrm{kg}$ en $40^{\mathrm{e}}$ semaine; la quantité de matières grasses sécrétée, maximum (940 g) dès la $2^{\mathrm{e}}$ semaine, diminue assez rapidement au cours des 3 premiers mois ( $-390 \mathrm{~g}$ ) et ensuite plus lentement ( $-380 \mathrm{~g}$ au cours des 7 derniers mois).

Sécrétion des acides gras des matières grasses du lait.

La composition en acides gras des matières grasses du lait présente une évolution importante au cours des deux premiers mois de la lactation, puis demeure relativement stable par la suite (tabl. 2).

La proportion d'acide butyrique $\left(\mathrm{C}_{1}\right)$ et celle du composant indéterminé $(\mathbf{I})$ qui apparaît en tête de chromatogramme, sont maximum dès la première semaine de lactation et diminuent régulièrement tout au long de la lactation.

La proportion des acides gras courts dont les chaînes saturées comprennent 6 à $I_{4}$ atomes de carbone, augmente au cours des deux premiers mois puis, après une phase maximale assez stable, diminue très légèrement au cours de la $2^{\mathbf{e}}$ moitié de la lactation. L'amplitude et la durée de l'accroissement sont d'autant plus grandes que la chaîne carbonée est plus longue $(+0,6$ point et 4 semaines pour les acides caproïque $\left(\mathrm{C}_{6}\right)$ et caprylique $\left(\mathrm{C}_{8}\right),+\mathrm{I}, 8$ points et 8 semaines pour le caprique $\left(\mathrm{C}_{10}\right)$, $+2,6$ points et +6 points et $I_{4}$ semaines pour le laurique $\left(\mathrm{C}_{12}\right)$ et le myristique $\left(\mathrm{C}_{14}\right)$ respectivement). Le rapport laurique sur caprique $\left(\mathrm{C}_{12} / \mathrm{C}_{10}\right)$ augmente durant la lactation passant de $I, 22$ en première semaine à $I, 37$ au $4^{\mathrm{e}}$ mois, puis à $I, 48$ au $9^{\mathrm{e}}$ mois.

La proportion des acides gras longs (stéarique, oléique, linoléique, linolénique) diminue brutalement au cours de la première partie de la lactation, puis augmente très légèrement par la suite. On observe la valeur minimum pour l'acide stéarique à la $34^{\mathrm{e}}$ semaine et pour l'acide oléique à la $\mathrm{I} 4^{\mathrm{e}}$ semaine.

I a proportion d'acide palmitique, constituant le plus important, présente tune évolution de moindre amplitude : minimum après le vêlage, elle augmente lentement mais régulièrement au cours de la première moitié de la lactation $(+6$ points $)$ puis se stabilise par la suite.

Les proportions d'acides gras mineurs à chaîne ramifiée ou non à I4 ou I 5 atomes de carbone ne cessent d'augmenter durant toute la lactation, leurs valeurs triplant environ; celles des acides gras mineurs à chaîne saturée ou non, ramifiée ou non à I6 ou 17 atomes de carbone diminuent au début de la lactation, puis augmentent pour atteindre leur maximum au tarissement.

D'une manière générale, la proportion des acides gras insaturés diminue au cours des 3 mois qui suivent le vêlage et varie peu ensuite.

$\mathrm{Si}$ on exprime les résultats en grammes d'acides gras par kilogramme de lait, on remarque que les teneurs en acides gras courts sont sensiblement constantes au cours de la lactation, variant de part et d'autre de $\mathrm{I} o \mathrm{~g} / \mathrm{kg}$; les teneurs en acides gras longs sont maximum après le vêlage $(20 \mathrm{~g} / \mathrm{kg})$ et diminuent au cours des 15 premières semaines, passent par un minimum ( $9 \mathrm{~g} / \mathrm{kg}$ ), puis augmentent jusqu'au tarissement (I2 $\mathrm{g} / \mathrm{kg}$ ) ; les teneurs en acide palmitique présentent une évolution comparable, 


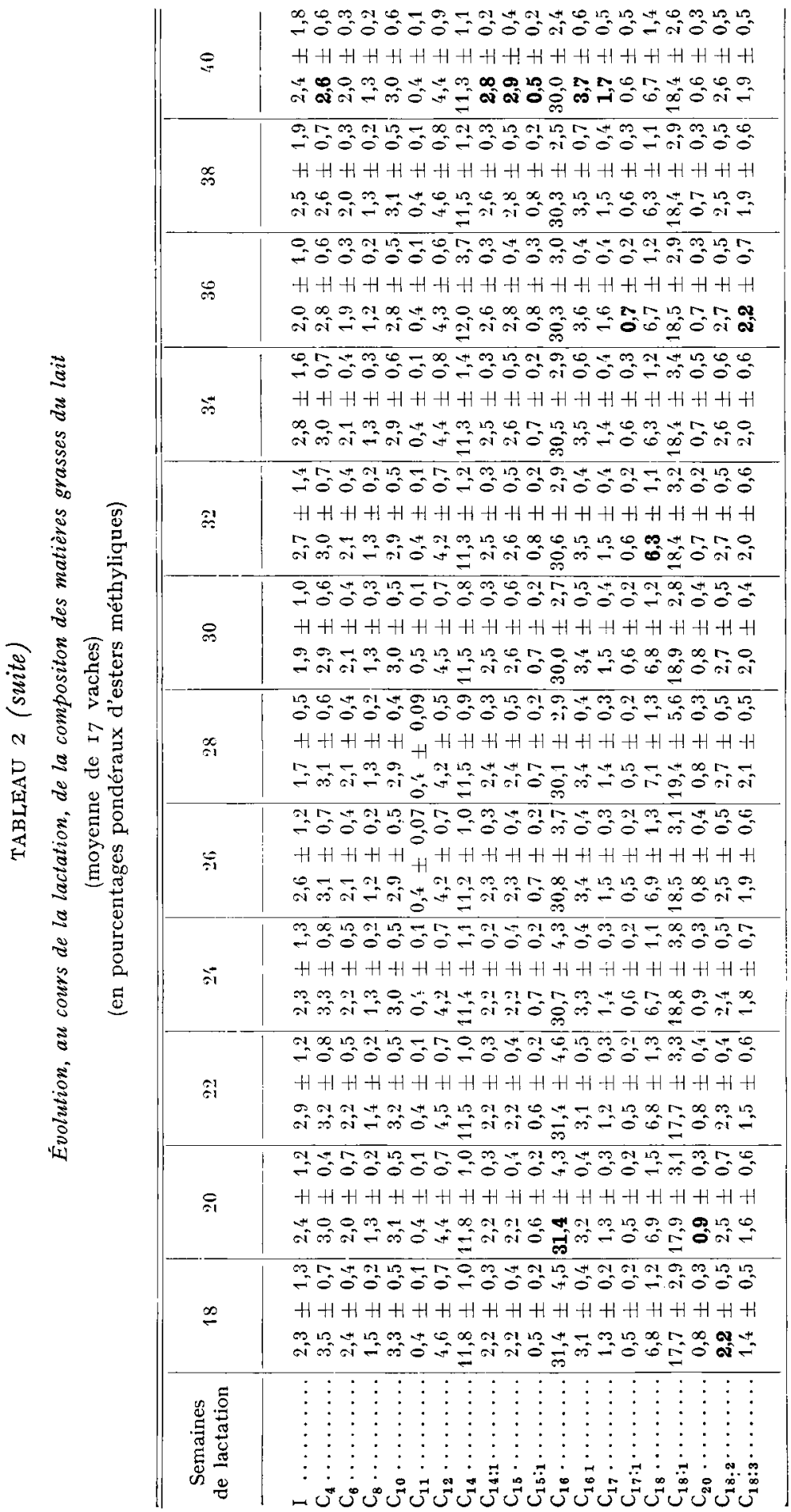

Annales de Biologie animale. - I970. 


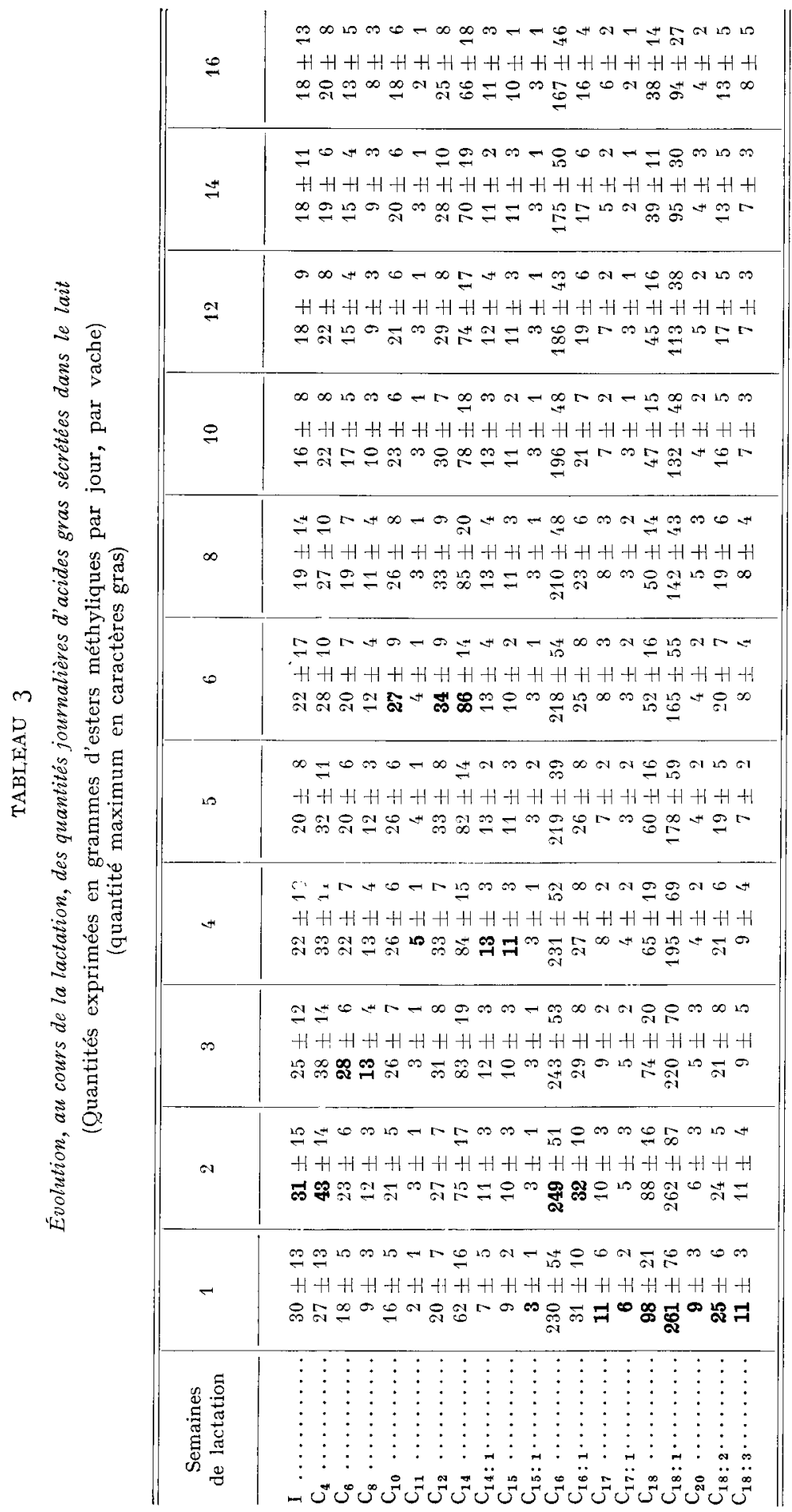




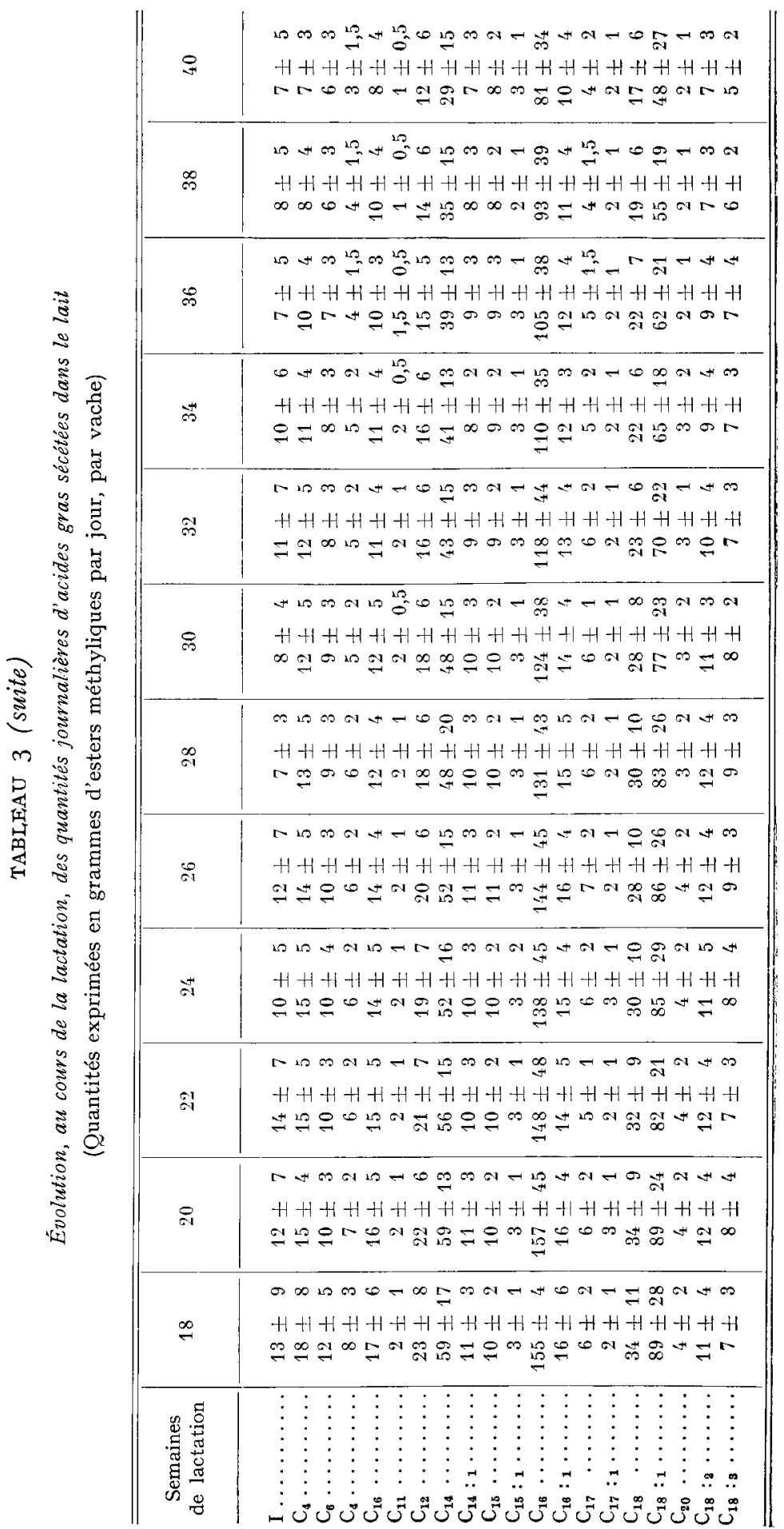


maximum après vêlage ( $\mathrm{I} 2 \mathrm{~g} / \mathrm{kg})$, elles sont minimales $(9 \mathrm{~g} / \mathrm{kg})$ à la $2^{\mathrm{e}}$ semaine et remontent à $\mathrm{I} 2 \mathrm{~g} / \mathrm{kg}$ à la fin de la lactation.

Les quantités d'acides gras sécrétées varient différemment en fonction du stade de lactation, selon la nature de ces acides (tab1. 3).

La quantité d'acides gras courts augmente dans les premières semaines qui suivent la mise bas. L'augmentation est d'autant plus longue et importante que la chaîne carbonée est plus longue : 2 semaines et +75 p. Ioo pour l'acide butyrique, 3 semaines et 40,45 et 60 p. Ioo respectivement pour le caproïque, le caprique et le caprylique, 6 semaines et $35 \mathrm{p}$. Ioo pour le laurique et le myristique. A partir du $2^{\mathrm{e}}$ mois de lactation les quantités sécrétées diminuent de I 5 à Io p. Ioo par mois, selon la longueur de la chaîne et le mois de lactation ; inversement les quantités sécrétées d'acides stéarique et oléique, qui sont maximum dès la semaine du vêlage, diminuent très rapidement au cours des 8 premières semaines (-30 $\mathrm{p}$. Ioo par mois), puis beaucoup plus lentement (一 II p. Ioo par mois) .

La quantité d'acide palmitique, maximale dès la $2^{\mathrm{e}}$ semaine, diminue ensuite régulièrement de II p. roo par mois tout au long de la lactation.

Les quantités journalières d'acides gras mineurs en $C_{14}$ et $C_{11}$ varient peu au cours de la lactation, mais elles ont tendance à diminuer très légèrement.

\section{Comparaison entre les deux lots - Différences individuelles}

Sur l'ensemble de la lactation, les vaches du lot I (Jouy) produisent significativement plus de lait et de matières grasses $(\mathrm{P}<0,05)$ que celles du lot II (La Minière) ; mais ce lait présente un taux butyreux plus faible et ces matières grasses sont légèrement plus riches en acides gras courts $\left(\right.$ de $\left._{4} \mathrm{à}_{12}\right)$ et en acides gras longs $\left(\mathrm{C}_{18}\right.$ saturés et insaturés), mais plus pauvres en acides gras moyens $\left(C_{14}\right.$ et $\left.C_{16}\right)$. Ainsi, en moyenne, les vaches du lot I qui produisent plus de matières grasses sécrètent autant d'acide palmitique que celles du lot II, un peu plus de myristique et beaucoup plus d'acides stéarique et oléique et d'acides gras courts (tab1. 4). Par ailleurs, on remarque que les animaux du lot I ont consommé moins de foin long ( $I, I$ tonne au lieu de $I, 7)$, un peu plus de fơin condensé $(2,95$ tonnes au lieu de 2,65$)$ et beaucoup plus de concentré (970 kg au lieu de 5 I $5 \mathrm{~kg}$ ).

Si le sens et l'amplitude des variations de chacune des variables étudiées sont en général semblables dans les deux lots, on remarque cependant certaines différences (fig. I). La quantité de matière sèche ingérée maximum est du même ordre ( $9 \mathrm{~kg}$ ), mais les animaux du lot II ayant reçu leur foin condensé en quantité limitée à partir de la $I 9^{e}$ semaine en moyenne, présentent en fin de lactation un niveau d'ingestion moins élevé.

Les vaches du lot $I$, vêlant en février-mars, ont une production laitière mieux soutenue au cours des 3 premiers mois de la lactation que celles du lot II vêlant en novembre : accroissement initial de la quantité de lait plus élevé, durée de la phase ascendante plus longue, taux butyreux après vêlage plus élevé. En revanche, à partir du $3^{\mathbf{e}}$ mois de la lactation, la production laitière des vaches se maintient mieux chez les vaches du lot II. Les vaches du lot I ont présenté un bilan énergétique plus déséquilibré après vêlage et des pertes de poids vif plus importantes (- $40 \mathrm{~kg}$ au lieu de $-22 \mathrm{~kg}$ ). A partir du $3^{\mathrm{e}}$ mois de lactation, la production laitière se maintient mieux 


\section{TABLEAU 4}

Composition moyenne en acides gras des triglycérides du lait et quantitées totales sécrétées d'acides gras durant toute la lactation

\begin{tabular}{|c|c|c|c|c|c|c|}
\hline & \multicolumn{3}{|c|}{$\begin{array}{l}\text { Proportions d'acides gras } \\
\text { (p. } 100 \text { esters méthyliques) }\end{array}$} & \multicolumn{3}{|c|}{$\begin{array}{c}\text { Quantités totales d'acides gras sécrétées } \\
\text { (kg d'esters méthyliques) }\end{array}$} \\
\hline & $\begin{array}{c}\text { Lot I } \\
\text { (9 vaches) }\end{array}$ & $\begin{array}{c}\text { Lot II } \\
\text { (8 vaches) }\end{array}$ & $\begin{array}{l}\text { Ensemble } \\
\text { (17 vaches) }\end{array}$ & $\begin{array}{c}\text { Lot } I \\
\text { (9 vaches) }\end{array}$ & $\begin{array}{c}\text { Lot II } \\
\text { (8 vaches) }\end{array}$ & $\begin{array}{l}\text { Ensemble } \\
\text { (17 vaches) }\end{array}$ \\
\hline I (?) & $\underset{* * *}{3,17} \pm 0,03$ & $2,09 \pm 0,02$ & $2,66 \pm 0,06$ & $\begin{aligned} 4,96 \pm \underset{* * *}{0,76} & \end{aligned}$ & $2,85 \pm 0,86$ & $3,96 \pm 1,34$ \\
\hline $\mathrm{C}_{4}$ & $\underset{* * *}{3,76} \pm 0,02$ & $3,17 \pm 0,03$ & $3,48 \pm 0,04$ & $\begin{array}{c}6,08 \pm \underset{* * *}{0,29} \\
* 0\end{array}$ & $4,27 \pm 1,13$ & $5,23 \pm 1,21$ \\
\hline $\mathrm{C}_{6}$ & $\underset{* * *}{2,44} \pm 0,003$ & $2,16 \pm 0,03$ & $2,30 \pm 0,02$ & $3,87 \pm \underset{* * *}{0,81}$ & $2,93 \pm 0,90$ & $3,42 \pm 0,96$ \\
\hline $\mathrm{C}_{8}$ & $\underset{* * *}{1,47} \pm 0,003$ & $1,29 \pm 0,004$ & $1,39 \pm 0,01$ & $2,34 \pm \underset{* * *}{0,52}$ & $1,75 \pm 0,51$ & $2,06 \pm 0,58$ \\
\hline $\mathrm{C}_{10}$ & $\underset{* * *}{3,14} \pm 0,03$ & $3,06 \pm 0,0^{\prime}$ & $3,10 \pm 0,03$ & 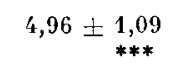 & $4,15 \pm 1,20$ & $4,58 \pm 1,18$ \\
\hline $\mathrm{C}_{11}$ & $\underset{* * *}{0,42} \pm 0,002$ & $0,38 \pm 0,001$ & $0,42 \pm 0,01$ & $0,7^{\prime} \pm \underset{* * *}{0,19}$ & $0,51 \pm 0,17$ & $0,63 \pm 0,21$ \\
\hline $\mathrm{C}_{12}$ & $\underset{* * *}{4,35} \pm 0,14$ & $4,13 \pm 0,16$ & $4,25 \pm 0,05$ & 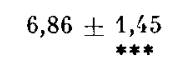 & $5,58 \pm 1,55$ & $6,26 \pm 1,60$ \\
\hline $\mathrm{C}_{14}$ & $\begin{array}{l}10,88 \pm 0,04 \\
* * *\end{array}$ & $11,43 \pm 0,22$ & $11,14 \pm 0,06$ & $\begin{array}{c}17,22 \pm \underset{\varnothing}{3,49} \\
\text {. }\end{array}$ & $15,45 \pm 3,97$ & $16,39 \pm 3,72$ \\
\hline$\Sigma C_{15}$ & $\underset{* * *}{4,37} \pm 0,16$ & $4,78 \pm 0,03$ & $4,58 \pm 0,02$ & $\begin{array}{c}6,82 \pm 0,95 \\
\varnothing\end{array}$ & $6,38 \pm 1,32$ & $6,62 \pm 1,13$ \\
\hline $\mathrm{C}_{16}$ & $\begin{array}{l}27,87 \pm 0,27 \\
* * *\end{array}$ & $31,85 \pm 2,37$ & $29,74 \pm 2,64$ & 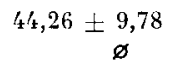 & $43,17 \pm 13,09$ & $43,75 \pm 11,09$ \\
\hline $\mathrm{C}_{18-1}$ & $\underset{* * *}{3,24} \pm 0,01$ & $3,33 \pm 0,02$ & $3,28 \pm 0,02$ & $\begin{array}{c}5,15 \pm \underset{*}{1,19} \\
\text {. }\end{array}$ & $4,49 \pm 1,11$ & $4,84 \pm 1,17$ \\
\hline $\mathrm{C}_{17}$ & $\underset{* * *}{1,64} \pm 0,003$ & $1,86 \pm 0,003$ & $1,75 \pm 0,01$ & $\begin{array}{c}2,60 \pm 0,46 \\
\varnothing\end{array}$ & $2,49 \pm 0,52$ & $2,54 \pm 0,47$ \\
\hline $\mathrm{C}_{18}$ & $\underset{* * *}{7,70} \pm 0,19$ & $6,80 \pm 0,29$ & $7,28 \pm 0,09$ & $12,10 \pm \underset{* * *}{2,13}$ & $9,00 \pm 1,61$ & $10,6^{4} \pm 2,44$ \\
\hline$C_{18-1}$ & $\begin{array}{l}20,69 \pm 1,64 \\
* * *\end{array}$ & $19,19 \pm 2,62$ & $20,00 \pm 2,22$ & $\underset{* * *}{32,95 \pm \underset{*}{7,93}}$ & $25,60 \pm 5,99$ & $29,49 \pm 7,84$ \\
\hline $\mathrm{C}_{20}(?)$ & $\underset{* * *}{0,77} \pm 0,002$ & $0,63 \pm 0,002$ & $0,70 \pm 0,01$ & $\begin{array}{c}1,23 \pm \underset{* * *}{0,30} \\
* *\end{array}$ & $0,85 \pm 0,24$ & $1,05 \pm 0,33$ \\
\hline$C_{18-2}$ & $\underset{* * *}{2,53} \pm 0,02$ & $2,45 \pm 0,02$ & $2,49 \pm 0,02$ & 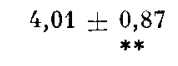 & $3,29 \pm \quad 0,81$ & $3,67 \pm 0,89$ \\
\hline $\mathrm{C}_{18-3}$ & $\underset{* * *}{1,51} \pm 0,02$ & $1,42 \pm 0,02$ & $1,46 \pm 0,09$ & $\underset{* * *}{2,37 \pm \underset{*}{0,46}}$ & $1,88 \pm 0,40$ & $2,14 \pm 0,49$ \\
\hline
\end{tabular}

Différences entre les lots $(\varnothing \mathrm{P}>0,05, * 0,05>\mathrm{P}>0,01, * * 0,01>\mathrm{P}>0,001, * * * \mathrm{P}<0,001)$. 
chez les vaches du lot II, bien qu'elles aient reçu leur foin condensé en quantité limitée à partir de la $\mathbf{r} g^{\mathbf{e}}$ semaine en moyenne. La remontée simultanée des teneurs du lait en matières grasses et en matières azotées est surtout nette à partir du mois d'août,
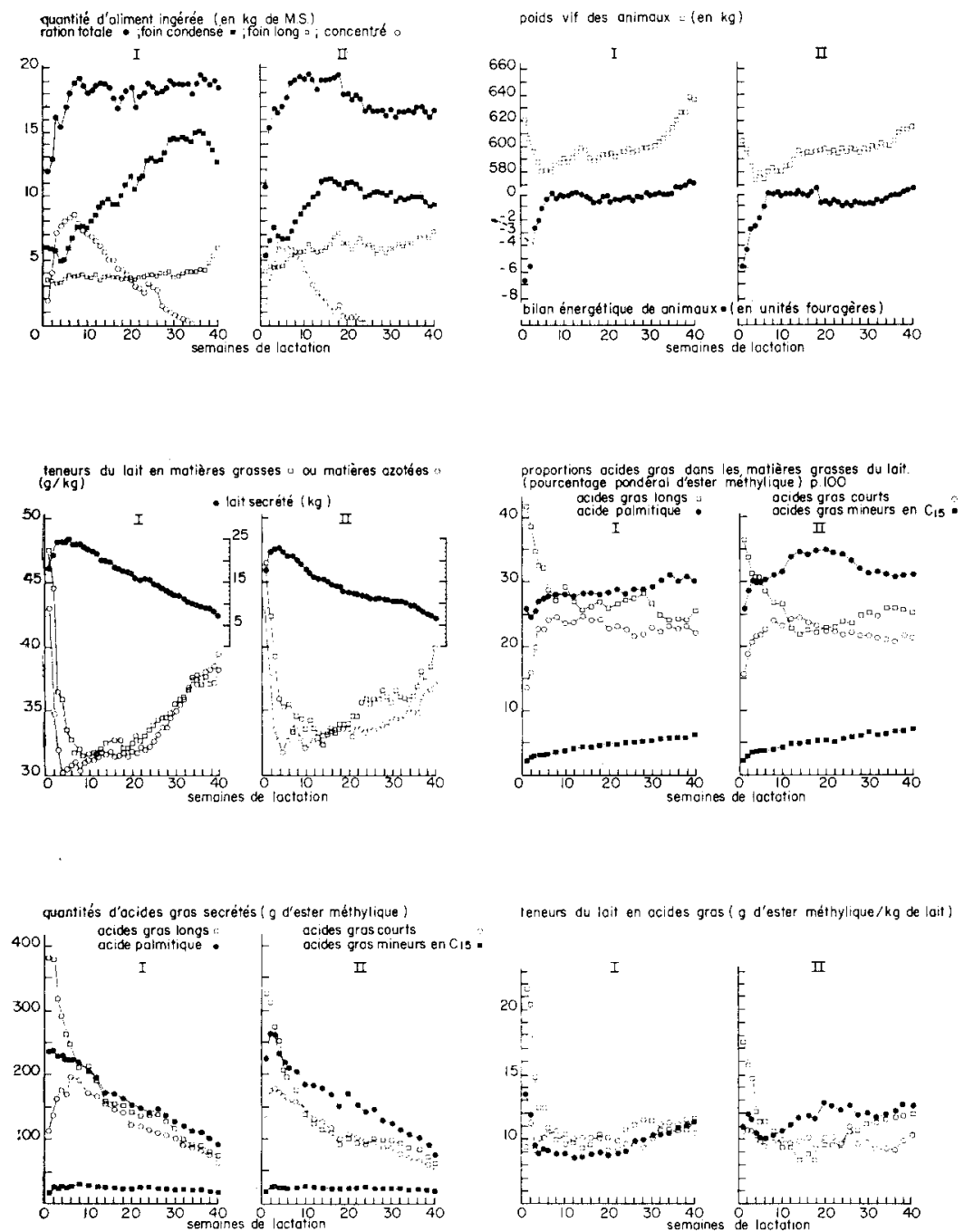

teneurs du loit en ocides gras ( $g$ d'ester méthylique/kg de lait)

FIG. 1. - Influence du stade de lactation pour 2 lots de vaches ayant vêlé en février à Jouy (I) et en novembre à la Minière (II) sur :

- les quantités d'aliments consommées, le bilan énergétique et le poids vif,

- les valeurs journalières des quantités de lait et des teneurs du lait en matières grasses et matières azotées,

- la sécrétion des acides gras des matières grasses du lait (composition, quantités sécrétées et teneurs du lait en acides gras).

c'est-à-dire à partir de la $20^{\mathrm{e}}$ semaine poutr le lot I et de la $35^{\mathrm{e}}$ semaine pour le lot II, alors que la composition en acides gras des triglycérides ne semble pas varier.

D'une manière générale on peut remarquer, dans chacun des lots, des variations simultanées du bilan énergétique, du taux butyreux et de la composition en acides 
gras des glycérides du lait. A des bilans énergétiques négatifs correspondent des valeurs plus élevées du taux butyreux (relativement à celle de la teneur en matières azotées, notamment) et de la proportion d'acides gras longs, et des valeurs plus faibles des proportions d'acides gras courts et d'acide palmitique. On observe ces variations plus particulièrement au cours des deux premiers mois de la lactation, mais aussi, de façon moins marquée, entre la $16^{\mathrm{e}}$ et la $28^{\mathrm{e}}$ semaine dans le lot $\mathrm{I}$ et entre la $20^{\mathrm{e}}$ et la $35^{\mathrm{e}}$ semaine dans le lot II.

Dans le cadre de cette expérience, à l'échelon de la lactation entière, les relations sont peu marquées entre les résultats de production laitière et la composition en acides gras des triglycérides ( 77 données). A des taux butyreux moyens élevés correspondraient des proportions d'acide palmitique élevées $(r=+0,49)$ et des proportions d'acides gras longs faibles $(r=-0,39)$; plus les vaches ont consommé de concentré, plus elles ont produit de lait $(r=+0,76)$ et de matières grasses $(r=+0,78)$ et plus les triglycérides de leur lait ont été riches en acides gras longs $\left(r=+0,5^{\circ}\right)$ et pauvres en acide palmitique $(r=-0,43)$ et en acides gras mineurs en $C_{\mathbf{1 4}}$ et $\mathbf{C}_{\mathbf{1 5}}$ $(r=-0,83)$.

Par ailleurs, au cours de la lactation d'une vache, le taux butyreux varie comme 1a teneur du lait en acides gras longs $(r=+0,76$ sur 39I données individuelles, valeurs individuelles de $+0,40$ à $+0,98$ ) (fig. 2).

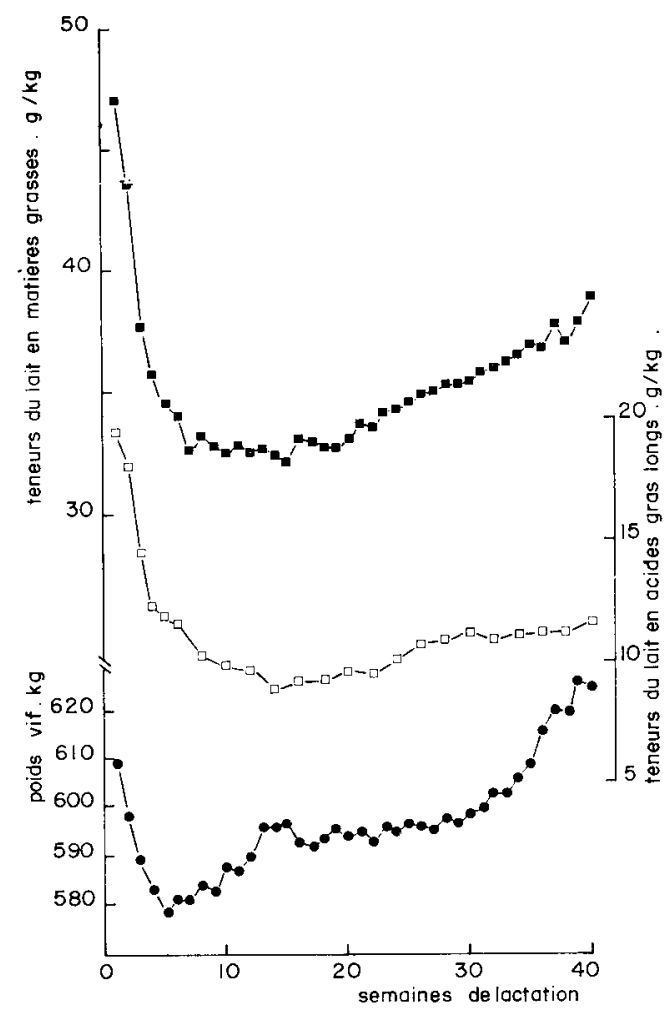

FIG. 2. - Évolution comparée, au cours de la lactation, du taux butyreux du lait . de la proportion d'acides gras longs dans les matières grasses du lait $\square$ et du poids vif • 
Enfin, au cours des six premières semaines de la lactation, les variations de la proportion d'acides gras longs des triglycérides du lait sont statistiquement liées à celles du déficit énergétique de la vache $(r=+0,7$ I, sur 102 données élémentaires).

\section{DISCUSSION}

\section{Critique des conditions expérimentales}

En dépit de notre volonté initiale, la ration que nous avons distribuée n'a pas été de nature constante sur toute la lactation : les quantités ingérées de concentré, maximum en $8^{\mathrm{e}}$ semaine, sont nulles avant la fin de la lactation, celles de foin condensé augmentent davantage que celles du foin long. Ce régime riche en éléments broyés et agglomérés a provoqué certaines perturbations digestives ou métaboliques au début de la lactation ( 3 dans le lot I, I dans le lot II) qui se sont traduites par des baisses passagères d'appétit et de production laitière ; certaines vaches $(2$ principalement) ont produit moins de lait qu'on pouvait le prévoir. Enfin, ce type de ration, qui est connu pour son effet dépressif sur le taux butyreux (cf. revue de VAN SoEST, I963), est certainement responsable des teneurs en matières grasses relativement faibles que nous observons. Cependant, la composition en acides gras des triglycérides du lait n'est pas sensiblement différente de celles observées sur des régimes hivernaux à base de foin long, d'ensilage, de betteraves et de concentré (cf. DECAEN et JoURNET, I967 ; DECAEN et Ghadaki, I970). Dans l'ensemble, la lactation s'est déroulée normalement : les niveaux moyens de production laitière et de teneur du lait en matières azotées ne sont pas inférieurs à ceux que l'on pouvait prévoir ; en fonction du stade de lactation, les quantités journalières de lait et de matières grasses, ainsi que les teneurs du lait en matières grasses et en matières azotées ont varié selon l'évolution habituellement décrite, notamment dans les deux mois qui suivent la parturition (Journe's et Jarrige, r960 ; DeCakn, Journeit et Poutous, r970). Il semble donc que nos conditions expérimentales peuvent être jugées, somme toute, suffisamment normales, pour permettre de proposer quelques conclusions généralisables.

\section{Influence du stade de lactation}

Nos résultats mettent en évidence deux phases dans l'évolution de la sécrétion des acides gras des matières grasses du lait au cours de la lactation.

Io Au cours des 6 à $\mathrm{I} 4$ premières semaines qui suivent la mise bas, la sécrétion des acides gras varie qualitativement et quantitativement : les quantités d'acides gras courts augmentent, celles d'acides gras longs diminuent rapidement ; la composition en acides gras des triglycérides varie.

$2^{\circ}$ Ensuite et jusqu'au tarissement, la composition en acides gras reste stable et seule la quantité de chacun des acides gras diminue régulièrement.

Nos résultats complets, en accord avec nos observations partielles présentées précédemment (DECAEN et ADDA, I966), confirment en partie ceux de STuLI et al. (r966), qui, rappelons-le, travaillèrent sur des vaches Holstein, vêlant en juillet, sous le climat chaud de l'Arizona et consommant du foin de luzerne long et un aliment 
concentré à base de tourteau de coton. Dans des conditions différentes des nôtres, ces auteurs trouvent que les proportions d'acides gras courts augmentent avec le stade de lactation et celles d'acides gras longs diminuent, cependant, les matières grasses du lait obtenu dans les semaines qui suivent le vêlage sont plus pauvres en acides gras courts et plus riches en acides gras longs que les nôtres et l'évolution de la composition est plus étalée dans le temps que calle que nous observons.

Nos résultats comme ceux de Srult et al. (1955) sont en accord avac les observations qui montrent que le colostrum et les laits des premiers jours de la lactation ont des matières grasses plus pauvres en acides gras courts et plus riches en acides gras longs (BALDWIN et Longenecker, I944; ANANTAKRISHNAN, BHALE RAO et Paul, I946; Hosogai, I963 et ADda, I964). Contrairement à celles de Stuli et al. (I966), nos observations confirment celles de KUHL,Man et GAL,LUP (I939) et KUzDzALSavore (I956) qui rapportent une diminution de l'indice d'iode des matières grasses du lait au cours des premiers mois de la lactation. F,nfin, nous mettons clairement en évidence que les modifications de la composition en acides gras, notamment au début de la lactation, sont liées à la diminution de la sécrétion de chacun des acides gras longs, mais aussi à l'augmentation des quantités sécrétées des acides gras courts. Nos obsarvations viennent à l'appui de la théorie proposée par POPJACK et al. (I95I) et exposée plus tard, entre autres, par GaRToN (I960-I963) ou Jones (I969) selon laquelle l'origine des acides gras du lait est au moins double; d'une part les acides courts $\left(\mathrm{C}_{4}\right.$ à $\left.\mathrm{C}_{16}\right)$ sont synthétisés par le tissu mammaire à partir de l'acétate et du $\beta$-hydroxybutyrate circulant dans le sang, d'autre part les acides gras longs (à partir de $C_{16}$ et peut-être $C_{14}$ ) seraient prélevés de formes circulant, préformées, dans le sang.

L'évolution de la sécrétion des acides gras des triglycérides du lait au cours du premier quart de la lactation est un phénomène général. Si la nature de la ration agit sur la composition en acides gras des matières grassas (BRowN, Stul, et Stotr, I962 ; DelaGe et FEHR, I967), nos résultats, ceux de STul, et al. (I955) et de DECAFN et JouRnet ( $19^{67}$ ) montrent que cette évolution a lieu, d'une manièra générale, sur des rations hivernales de différents types. L'un d'entre nous a montré (DECAEN et JoURNET, I 967) que cette évolution de la composition en acides gras des matières grasses du lait et celle du taux butyreux, au cours des 6 premières semaines de la lactation, peuvent s'expliquer, en partie, par la mobilisation des réserves lipidiques, provoquée par un bilan énergétique négatif et libérant des acides gras libres dans le sang. Dans les semaines qui suivent la mise bas, l'activité mammaire sa met en route ; les quantités sécrétées de lait, de lactose, de matières azotées (cıséine, surtout) augmentent, mais aussi celles d'acides gras courts. Ce phénomène est progressif, comme en témoigne le fait que la quantité maximum journalière d'acides gras courts sécrétée est atteinte d'autant plus tard que la chaîne carbonée est plus longue. Il correspondrait moins à un accroissement du nombre de cellules sécrétrices, qui sarait fixé dans les jours qui suivent la mise bas (DENAMUR, Ig69) qu'à une augmentation de l'activité sécrétrice de ces cellules, qui est, en grande partie bloquée jusqu'à la mise bas et pourrait être limitée, durant les premières semaines de lactation, par des mécanismes de régulation métabolique (BALDWIN, I969). Juste après le vêlage, le déclenchement de lactation, provoquant une élévation brutale des besoins, place l'animal en bilan énergétique déficitaire et celui-ci réagit en mobilisant ses réserves corporelles : la vache sécrète alors des matières grasses de même composition, c'est-à-dire pauvres 
en acides gras courts et riches en acides gras longs, que si elle était sous-alimentée expérimentalement (SMith et Dastur, I938; Luick et Smith, i963; Decean et ADDA, I970). Au cours des deux premiers mois de la lactation, la vache augmente son niveau de consommation et atteint son équilibre énergétique : l'accroissement des apports des précurseurs sanguins (acétate, $\beta$-hydroxybutyrate) pourrait être en grande partie responsable de l'augmentation de la sécrétion des acides gras courts, la baisse de l'intensité de mobilisation des réserves corporelles entraînant la diminution de la sécrétion des acides gras longs. Par ailleurs, la même évolution a été observée chez la Truie, la mobilisation des réserves corporelles se faisant au profit de la sécrétion des acides gras des matières grasses du lait, de façon décroissante et préférentiellement à partir des acides gras longs (SALMON-LEGAGNEUR, Ig63, I964, I965).

Au cours des trois derniers quarts de la lactation, bien que le taux butyreux augmente très nettement, la composition des matières grasses du lait reste stable; la sécrétion du lait et du lactose diminuerait plus rapidement que celles des matières grasses et des matières azotées, d'où l'élévation des teneurs du lait en ces constituants.

Au cours de la lactation nous observons une élévation continue des proportions d'acides gras mineurs en $\mathrm{C}_{14}$ en $\mathrm{C}_{15}$. Ces acides gras mineurs, dont la chaîne carbonée compte un nombre impair d'atomes de carbone ou bien est ramifiée, seraient synthétisés par l'intermédiaire des microorganismes du rumen à partir d'acides gras volatils issus de la digestion, notamment de la désamination des matières azotées des aliments (EI, ShazLy, I952 ; ANNISON, I954). Il est possible que l'augmentation des quantités consommées de foin de luzerne entraîne un apport important de protéines alimentaires dont l'excès serait à l'origine des produits de désamination. La part des acides gras mineurs augmenterait au cours de la lactation, alors que les quantités totales d'acides gras sécrétées diminuent.

D'une manière générale, la diminution du taux butyreux au début de la lactation, serait provoquée par une baisse de sécrétion mammaire des acides gras longs, qui serait liée, semble-t-il, à une baisse d'intensité de la mobilisation des réserves corporelles ; en revanche, l'augmentation du taux butyreux, en fin de lactation, n'est pas associée à une variation de la composition des acides gras des triglycérides du lait, mais proviendrait plutôt d'une diminution de la sécrétion des matières grasses moins rapide que celle du lait et du lactose.

\section{Influence de la saison de vêlage}

Afin d'étudier l'influence du mois de vêlage, nous avions constitué deux lots de vaches ( $\mathbf{I}$ et $\mathbf{I I}$ ). Cependant les différences entre ces deux lots portent non seulement sur les dates de vêlage, mais également sur d'autres points : niveau moyen de production laitière et de taux butyreux, troupeaux, races, rationnement (les quantités de foin condensé furent limitées pour des raisons matérielles, à $\mathrm{I} 2 \mathrm{~kg}$ par vache à partir du mois de mars dans le lot II). Ces différences rendent difficile l'interprétation des résultats et la mise en évidence de l'influence de la saison de vêlage. On peut toutefois proposer les remarques suivantes :

Io Après les vêlages de février-mars, au début de la lactation, la sécrétion du lait en général et celle des acides gras des triglycérides en particulier semblent plus stimulées qu'après les vêlages de novembre : les vaches sont plus sous-alimentées et 
perdent davantage de poids. Ceci est en accord avec des observations plus générales sur les variations saisonnières de la production laitière (DECAEN, CALOMITI et Pourous, I970 $a$ et $b$ ) et du poids vif (SchulTz et DAvis, I96I).

$2^{\circ}$ La saison la plus favorable au maintien et au développement de la production laitière semble être janvier à mai, ce qui expliquerait la meilleure persistance observée après les vêlages d'automne. La saison la plus défavorable serait l'été : la remontée des teneurs du lait en matières grasses et matières azotées, observée en août et septembre, viendrait d'une baisse de la sécrétion du lait plus rapide que celle des matières grasses et matières azotées. Il est possible que les différences entre nos observations et celles de STULL et al. (Ig66) aient pour origine la différence de saison de vêlage, d'autant plus qu'en Arizona l'effet dépressif de l'été est accentué par les fortes chaleurs (RICHARDSON et al., Ig6r).

Reçu pour publication en mai 1970.

\title{
SUMMARY
}

\author{
CHANGES IN SECRETION OF FATTY ACIOS \\ OF MIL, FAT DURING LACTATION IN COWS
}

The influence was studied of stage of lactation on major fatty acid composition of cow's milk fat (table 2) and on amounts of the fatty acids secreted (table 3 ).

The composition was estimated by gas-chrcmatography of the methyl and butyl esters. These esters were prepared by transesterificaticn of individual samples of milk fat collected once a week during the first 6 weeks of lactation then once every 15 days until drying off.

For the trial 77 cows were fed individually during $4^{\circ}$ weeks of lactation on long lucerne hay, ground and pelleted lucerne hay and a concentrate feed given according to requirements. The I7 cows were in 2 groups : one group calves in winter at Jouy and the other in autumn at La Minière. Characteristics of the cows are shown in table I. For each cow, in addition to secretion of fatty acids, intake of feed, yield of milk and its fat and protein contents, liveweight and energy balance were recorded (fig. I).

During the first 6 to Io weeks of lactation the fatty acid composition of the triglycerides changcd systematically (table 2 ). The propcrtions of short-chain fatty acids $\left(C_{4}\right.$ to $\left.C_{14}\right)$ increased, that of palmitic acid changes little and those of leng-chain fatty acid $\left(\mathrm{C}_{18}\right)$ decreased. These changes were the result of a quantitative increase in secretion of short-chain fatty acids and a decrease in that of the long-chain fatty acids (table 3). During this period milk yield increased, fat and protein contents decrease $d$ and intake of feed increased. The energy balance of the cows, strongly negative after calving, improved progressively to reach equilibrium, the cows losing weight (fig. I).

A correlation coefficient between energy deficit and proportion of long-chain fatty acids in milk fat $(+0.7 \mathrm{I})$ was calculat $\epsilon d$ for 102 results. The fall in fat content of milk se $\epsilon \mathrm{n}$ during the first 6 or 7 weeks of lactation would result from decrease in secretion of long-chain fatty acids, related to a decrease in intensity of mobilization of body reserves (fig. 2).

After the third month of lactation yields of milk and of fat decreased and fat content increased. Composition of the major fatty acids in milk fat did not change (table 2). The proportion of minor fatty acids, branched-chain or unsaturated at $\mathrm{C}_{14}$ and $\mathrm{C}_{15}$ continued to increase up to drying off (fig. I).

For the whole lactation changes in fat content were significantly related to those in the content of long-chain fatty acids in milk: $r=0.76$ for $38 \mathrm{r}$ results (fig. 2). 


\section{RÉFÉRENCES BIBLIOGRAPHIQUES}

AdDA J., I964. Die Fettesauren der Triglyceride in der kolostralmilch der Kuh. Kieler Milchwirtschafftliche Forchuns gberichte, 16, 379-387.

Annatakrishnan C. P., Bhale Rao V. R., Paul T. M., I946. The components acids of cow colostrum fat. Biochem, J., 40, 292-297.

Annison E. F., 1954. Some observations on volatile fatty acids in the sheep rumen. Biochem. J., 57, $400-404$.

Baldwin A. R., Longnecker H. P., i944. Component fatty acids from the fatof cow colostrum J. Biol. Chem., 155, 407.

Baldwin R. L., 1969. Development of milk synthesis. J. Dairy Sci, 52, 729-736.

Brown W. H., Stull J. W., Stott G. H., I962. Fatty acid composition of milk. II. Effect of roughage and dietary fat. J. Dairy Sci., 45, I9r-I96.

Clément G., Bezard J., ig6r. Technique de dosage par chromatographie gaz-liquide d'un mélange d'acides gras, du butanoíque au docosanoïque. C. R.s Acad. Sci., 253, 56I-566.

Decaen C. AdDa J., Ig66. Évolution de la sécrétion des acides gras des glycérides du lait de vache, au cours de la lactation. XVII Congr. Intern. Laiterie, A1, I6I-I 7 I.

DEcaEn C., Adda J., I970. Influence d'une sous-alimentation passagère sur la sécrétion des acides gras des glycérides du lait et sur les concentrations du sang en acides gras libres et en triglycérides chez la vache (en préparation).

Decaen C., Ghadaki M. B., I970. Influence de la mise à l'herbe, de la nature de l'herbe et de son stade de végétation sur la sécrétion des acides gras des matières grasses du lait. Ann. Zootech. (sous presse).

Decaen C., Journet M., Poutous M., r97o. Evolution de la production laitière de la vache au cours des deux premiers mois de la lactation. I. Description graphique de l'évolution journalière de la quantité de lait sécrétée, du taux butyreux et de la quantité de matières grasses sécrétée. Ann. Zootech. (sous presse).

Decaen C., Calomitis. Poutous M., ig7o a. Évolution de la production laitière de la vache au cours des deux premiers mois de la lactation. II. Analyse des variations de la quantité de lait. Ann. Zootech. (sous presse).

Decaen C., Calomiti S., Poutous M., I970 $b$. Évolution de la production laitière de la Vache au cours des deux premiers mois de la lactation. III. Analyse des variations de la quantité de matières grasses et du taux butyreux. Ann. Zootech. (en préparation).

Decaen C., Journet M., I966. Influence saisonnière sur la production et la composition du lait. Ann. Zootech., 15, 259-277.

Decaen C., Journet M., I967. Évolution, au début de la lactation, de la sécrétion des principaux acides gras du lait et de la concentration en acides gras libres du sang chez la Vache. Ann. Biol.

anim. Bioch. Biophys., $7, \mathrm{I} 3 \mathrm{I}-143$.

Dr.age J., Fehr P. M., I967. Influence des lipides alimentaires sur la sécrétion des acides gras par la mamelle de la Chèvre. Influence de la teneur du régime en lipides sur le taux butyreux du lait et sa composition en acides gras. Ann. Biol. Anim. Bioch. Biophys., 7, 437-444.

Denamur R., 1969. Les acides désoxyribonucléiques au cours de la lactogenèse. Ann. Biol. anim. Bioch. Biophys., 9, 287-296.

El ShazLY K., I952. Dégradation of protein in rumen of the sheep. I. Some volatile fatty acids, including branched-chain isomers formed in vivo. Biochem., J., 51, 640-647.

Garton G. A,, 1960. Lipid metabolism in herbivorous animals. Nutr. Abst. Rev., 30, I-I6.

Garton G. A., 1963. The composition and biosynthesis of milk lipids. J. Lip. Res., 4, 237-253.

JARRIGE R., JOURNET M., I959. Influence des facteurs alimentaires et climatiques sur la teneur en matières grasses du lait. Ann. Nutr. anim., 13, $133^{-277 .}$

Jones E. A., I969. Reviews of the progress dairy science. Section A : recent developments in the biochemistry of the mammary gland. J. Dairy Res., 36, I45-I67.

Journet M., JARrige R., I960. Évolution de la sécrétion des matières grasses, des matières azotées et du lactose au cours du premier mois de lactation. Ann. Zootech., 9, 133-I53.

Kuhlman A. H., Gallup W. D., 1939. The effect of ration on butterfat constants. J.Dairy Sci., 22, 424 .

Kuzdal-Savoie S., I956. Influence du stade de lactation sur les caractéristiques des matières grasses du beurre. Ann. Tech. agric., 5, ז-30.

Luick J. R., Smith L., I963. Fatty acid synthesis during fasting and bovine ketosis. J. Dairy Sci., 46, I 25 I-I 255 . 
Matrson S., r962. Lipids in milk. Intern. Dairy Fed. Bull. 1962. Part II, I-I6.

PoPJACK G., French T. H., Folley S. J., I95I. Utilization of acetate for milk fat synthesis in the lactating goat. Arch. Biochem., 23, 508-5 Io.

Posthumus S., 1960. The determination of the protein content of milk by the colorimetric method. Neth. milk Dairy J., 14, 319-333.

Richardon C. W., Johnson H. D., Gehre C. W., Goerlitz D. F., ig6r. Effects of environmental temperature and humidity on the fatty acid composition of milk fat. J. Dairy Sci., 54, 1937I940.

Salmon-Legagneur E., I963. Relation entre les graisses du régime, les lipides corporels et les lipides du lait chez la Truie. C. R. Acad. Sci. 256, I372-1 374 .

Salmon-Legagnelr E., I964. Relation entre les graisses ingérées, les lipides corporels et les acides gras du lait chez la Truie. Ann. Biol. anim. Bioch. Biophys., 4, I4I-I55.

Salmon-LEGAGNeUR E., I965. Quelques aspects des relations nutritionnelles entre la gestation et la lactation chez la Truie. Thèse Univ. Paris, I43 pages.

Schultz A. B., Davis H. P., I96r. Changes in body weight during the first pregnancy for Holstein heifers calving at different seasons. J. Dairy Sci, 44, I717-I720.

Sмith J. A. B., Dastur B. N., 1938. Studies in the secretion of milk fat. II. The effect of inanition on the yield and composition of milk fat. Bioch. J., 32, 1868-I876.

Stull J. W., Brown W. H., Carlos Valdez, Tucker H., ig66. Fatty acid composition of milk. III. Variation with stage of lactation. J. Dairy Sci., 49, I 40I-I405.

VAN Soest P. J., rg63. Ruminant fat metabolisin with particular reference to factors affecting low milk and feed efficiency. A review. J. Dairy Sci, 46, 204-2 I6. 